\title{
IMPROVED NUMERICAL MODEL OF THE ARTERIAL WALL APPLIED FOR SIMULATIONS OF STENT DEPLOYMENT WITHIN PATIENT-SPECIFIC CORONARY ARTERIES
}

Tijana Djukic

Bioengineering Research and Development Center, BiolRC,

Kragujevac, Serbia

University of Kragujevac,

Institute for Information

Technologies,

Kragujevac, Serbia

\section{Oberdan Parodi}

Institute of Clinical Physiology, National Research Council, Pisa, Italy

\section{Igor Saveljic}

Bioengineering Research and Development Center, BiolRC, Kragujevac, Serbia

University of Kragujevac, Institute for Information

Technologies,

Kragujevac, Serbia

\section{Nenad Filipovic}

Bioengineering Research and Development Center, BiolRC, Kragujevac, Serbia

University of Kragujevac, Faculty of Engineering, Department of Applied Mechanics and

Automatic Control,

Kragujevac, Serbia

\section{Gualtiero Pelosi}

Institute of Clinical Physiology, National Research Council, Pisa, Italy

Key words: stent expansion, prediction of shape, deformation, hyperelastic material model, stressstrain relationship, finite element method

\section{Cite article:}

Tijana, D., Igor, S., Gualtiero, P., Oberdan, P., \& Nenad, F. [2021]. Improved numerical model of the arterial wall applied for simulations of stent deployment within patient-specific coronary arteries.

Journal of Applied Engineering Science, 19(1), 109 - 113. DOI:10.5937/jaes0-27805

Online aceess of full paper is available at: www.engineeringscience.rs/browse-issues 


\title{
IMPROVED NUMERICAL MODEL OF THE ARTERIAL WALL APPLIED FOR SIMULATIONS OF STENT DEPLOYMENT WITHIN PATIENT-SPECIFIC CORONARY ARTERIES
}

\author{
Tijana Đukić1,2*, Igor Šaveljić1,2, Gualtiero Pelosi ${ }^{3}$, Oberdan Parodi ${ }^{3}$, Nenad Filipovićc ${ }^{1,4}$ \\ ${ }^{1}$ Bioengineering Research and Development Center, BiolRC, Kragujevac, Serbia \\ ${ }^{2}$ University of Kragujevac, Institute for Information Technologies, Kragujevac, Serbia \\ IInstitute of Clinical Physiology, National Research Council, Pisa, Italy \\ ${ }^{4}$ University of Kragujevac, Faculty of Engineering, Department of Applied Mechanics and \\ Automatic Control, Kragujevac, Serbia
}

Arterial stenosis is the obstruction of normal blood flow that is caused by atherosclerosis. One of the endovascular treatment procedures in this case is the implantation of a stent to restore the blood flow. This study presented an improved numerical model that can precisely simulate the deformation of human arterial wall in coronary arteries, during the stent deployment process. The new model considered the arterial wall as an incompressible, isotropic and hyperelastic material. The material coefficients were defined according to experimental values presented in literature. The accuracy of the numerical model was investigated by comparing the results with follow up data obtained in clinical examination. The small relative and standard deviation error prove that this numerical model can be used to assist clinicians in decision making and treatment planning with reliable predictions of the outcome of the stent deployment procedure.

Key words: stent expansion, prediction of shape, deformation, hyperelastic material model, stress-strain relationship, finite element method

\section{INTRODUCTION}

Atherosclerosis is a progressive disease characterized by the accumulation of lipids and fibrous elements in arteries. Atherosclerotic plaques can cause significant local changes of the vessels and obstruct blood flow. One of the most common treatments of atherosclerotic plaque is the implantation of a stent, that ensures that the anatomy of the specific lesion is restored to allow normal blood flow. Stent represents a small metal cylindrical device that pushes the arterial wall outwards during its expansion. During this process, the arterial wall suffers large displacements and deformations, and these phenomena, together with geometric nonlinearities, have to be included in the numerical model that can simulate this process.

Many authors in literature simulated the deployment of the stent within coronary arteries [1-3]. One of the main drawbacks of the cited numerical models is that they use simplified geometry of the artery and don't consider the changes during the entire deployment process. The study presented in [4] presents a numerical model that simulates the entire stent deployment process using clinical patient-specific geometrical data. However, in study [4] the arterial wall is assumed to be linearly elastic and the mechanical characteristics of the wall are considered to be constant. In order to perform more realistic and more accurate simulations, a choice of a more appropriate numerical model for the simulation of the behavior of the human arterial wall is very important. In this paper, a more complex numerical model is incorporated, that models the arterial wall as a hyperelastic material. The material coefficients are taken from literature [5], where the authors measured these coefficients experimentally for human coronary arteries. The patient-specific clinical data is used to perform numerical simulation of stent deployment using both the old simplified model and the new model. The obtained results for both models are compared. Furthermore, the advantages of the new method are shown quantitatively through the comparison of results with clinical follow up data.

\section{NUMERICAL MODEL}

The numerical model considers three segments: stent expansion, interaction between stent and arterial wall and the deformation of the arterial wall. In this study, the stent expansion and between stent and arterial wall is modelled in the same way like it was described in the previous version of the numerical model [4]. The simplex deformable model proposed by Larrabide et al. [6] is used for modeling the stent expansion. The interpolation of forces acting on each entity along the contact surface is employed to simulate the interaction between stent and arterial wall. This interpolation is perfomed using the Dirac delta function [7].

Arterial wall consists of a mesh of nodes. The following equation is used to model the motion of these nodes in time:

$$
\begin{aligned}
& \mathbf{x}_{i}^{t+1}=\mathbf{x}_{i}^{t}+(1-\gamma) \cdot\left(\mathbf{x}_{i}^{t}-\mathbf{x}_{i}^{t-1}\right)+ \\
& +\alpha \cdot \mathbf{f}^{a}{ }^{a} \operatorname{int}\left(\mathbf{x}_{i}^{t}\right)+\beta \cdot \mathbf{f}^{a}{ }_{\text {ext }}\left(\mathbf{x}_{i}^{t}\right)
\end{aligned}
$$


where coordinates of the $i$-th node are denoted by $\boldsymbol{x}_{i}$, and upper indices $t$ and $t+1$ denote the time step. The coefficients $\alpha$ and $\beta$ are weight coefficients that are used to control the influence of internal and external forces. The value of these coefficients is taken to be 0.018 . The value of the damping parameter $y$ is taken to be 0.5 . These values are defined according to data available in literature [8]. The external force $f_{\text {ext }}$ that causes the motion of the nodes of the arterial wall is interpolated from the surrounding stent nodes. On the other hand, the stent and the external force cause a deformation that induces an internal force $f_{\text {ext }}$. This force represents the resistance of the arterial wall to the deformation. The finite element method is used to calculate this force as follows:

$\mathbf{f}_{\text {int }}^{S}=\int_{V} \mathbf{B}^{T} \boldsymbol{\sigma} d V$

where the internal stress is denoted by $\delta$ and the matrix of derivatives of interpolation functions is denoted by $\boldsymbol{B}$. This matrix is defined for the particular finite element considered in the simulation, in this case for the tetrahedral element.

The deformations and displacements of nodes of arterial wall are considered to be large and hence geometric nonlinearity was considered. The updated Lagrange method was employed for modeling the behavior of the arterial wall [9].

The relation between stress and deformation can be defined using an appropriate constitutive relation (like the linearly-elastic relation used in the previous version of the numerical model of stent deployment [4]), or it can be defined using some other material function. In this study, the arterial wall is considered to be made of an incompressible and isotropic material [10] and hence the hyperelastic Mooney-Rivlin model is used to model the behavior of the arterial wall. The hyperelastic material function can in this case be defined as:

$$
\begin{aligned}
W & =C_{10}\left(\bar{I}_{1}-3\right)+C_{01}\left(\bar{I}_{2}-3\right)+ \\
& +C_{20}\left(\bar{I}_{1}-3\right)^{2}+C_{11}\left(\bar{I}_{1}-3\right)\left(\bar{I}_{2}-3\right)+C_{02}\left(\bar{I}_{2}-3\right)^{2}
\end{aligned}
$$

This material function from Eq. (3) is used to determine the equation that defines the relationship between stress and strain, that is then inserted in Eq. (2). In Eq. (3) $\bar{I}_{1}$ and $\bar{I}_{2}$ represent the first and second invariants of the Cauchy-Green strain tensor and the constants $C_{i j}$ are the material coefficients that should be defined for the particular material whose behavior is simulated. In this case, the material coefficients for the healthy and atherosclerotic coronary arteries are experimentally calculated in literature [5]. These values are listed in Table 1 and are used in this study to model the behavior of arterial wall during stent deployment.

Since the clinical data for the specific patient that was used in this study enabled only the extraction of geometry of the coronary artery, it is considered that the material characteristics vary from the ones that are calculated for healthy and atherosclerotic artery. Namely, for each element of the mesh, the diameter of the artery in that particular segment is calculated. Based on this information, a weight coefficient (with value varying between 0 and 1 ) is calculated that determines the exact material coefficients for that particular element. If the weight coefficient is closer to 1 , it means that the particular element belongs to the healthier tissue of the artery and vice versa. Fig. 1 shows the weight coefficients for the particular coronary artery that is used in simulations in this study.

Table 1: Hyperelastic material coefficients of the healthy and atherosclerotic artery [5]

\begin{tabular}{|c|c|c|}
\hline Coefficient & Healthy artery & Atherosclerotic artery \\
\hline $\mathrm{C}_{10}[\mathrm{MPa}]$ & 2.101 & 0.666 \\
\hline $\mathrm{C}_{01}$ & -2.091 & 0.087 \\
\hline $\mathrm{C}_{02}$ & -92.125 & -15.778 \\
\hline $\mathrm{C}_{20}$ & -58.245 & -80.191 \\
\hline $\mathrm{C}_{11}$ & 145.826 & 107.893 \\
\hline
\end{tabular}

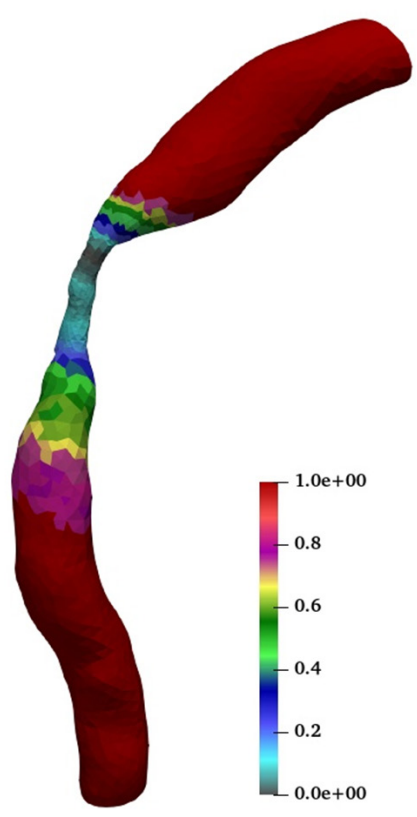

Figure 1: The weight coefficients of the arterial elements, determining whether the element belongs to fully healthy segment (value 1 on scale bar), fully atherosclerotic segment (value 0 on scale bar), or partially atherosclerotic segment

All the equations of all the three segments of the numerical model are solved iteratively. The condition to stop the simulation is to reach convergence. In this case, the maximum absolute change of the non-dimensional displacement of stent nodes is measured in each time step. When this change is less than $10^{-3}$, the convergence is considered to be reached. This value of the convergence criteria is chosen in accordance with previous simulations presented in literature [9]. 


\section{RESULTS AND DISCUSSION}

In this study, clinical data for a specific patient was obtained through the SMARTool project, that is funded under the European Programme Horizon 2020. The CT angiography (CTA) examination was performed on a clinical patient and included pre-operative and post-operative (follow up) examination. The available clinical data were CT scan DICOM images. The geometry of the coronary artery is extracted using the commercial software Mimics and the FEMAP software is then used to generate the finite element tetrahedral meshes.

In this Section, the benefits obtained by the introduction of the improved numerical model of the arterial wall will be discussed along with the presentation of the results.

The shapes of the artery obtained in numerical simulation of stent deployment are shown in Fig. 2. In Fig. 2A the initial shape of the artery is shown, while Fig. 2B and $2 \mathrm{C}$ show the shape of the artery obtained after the stent deployment simulation is performed. Fig. 2D shows the shape of the artery obtained by extracting the available follow up clinical CT scan images. As it can be observed, the shape of the artery obtained using the new and improved numerical model better resembles the results from follow up clinical examination. This can be further quantitatively proven by analyzing the areas of the lumen in specific cross sections. The blue and red lines shown in Fig. 2 actually represent the cross sections of the artery where the area of the lumen was calculated. The obtained values of areas are shown in a graph that plots these values for the chosen slices in Fig. 3. The overall relative error of the areas obtained in simulation using the previous version of the numerical model [4] compared with the follow up data is $12.45 \%$, while the same overall relative error of the areas obtained in simulation using the improved numerical model is only $4.41 \%$. The standard deviation error of the areas obtained using the improved numerical model is $1.54 \%$ compared to the follow up data.
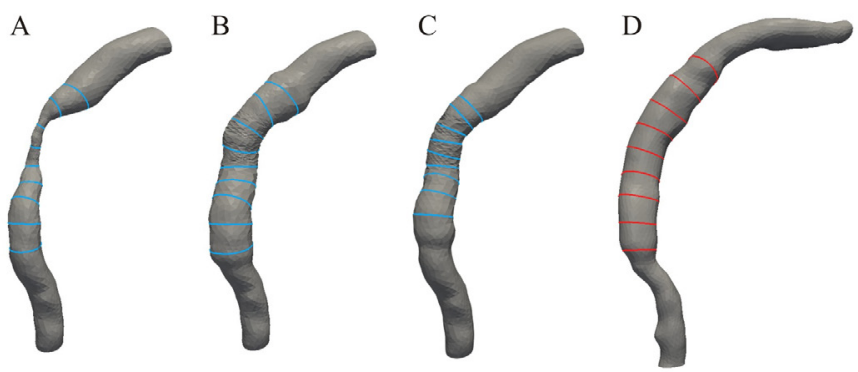

Figure 2: Results of the numerical simulation of stent deployment;

A - initial shape of the artery;

$B$ - shape of the artery after stent deployment using the simplified numerical model;

C - shape of the artery after stent deployment using the hyperelastic numerical model;

$D$ - shape of the artery obtained at the follow up clinical examination

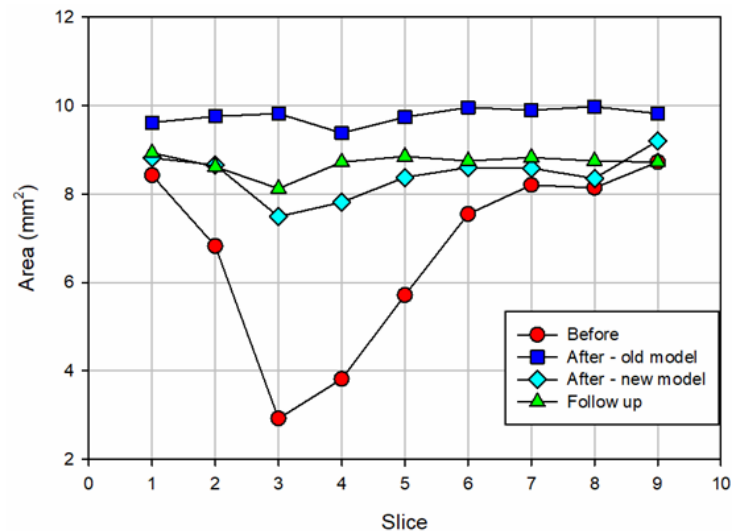

Figure 3: Values of area of the arterial lumen for the slices depicted in Fig. 2, for several considered moments- before and after stent deployment with old and new improved numerical model and from follow up examination

It is important to compare also other quantitative parameters that define the state of the arterial wall during and after numerical simulation. The distribution of the force that causes the deformation of the arterial wall, produced by the stent is shown in Fig. 4. It should be noted that this is the summed force for the whole simulation. From Fig. 4 it is possible to observe the differences of the results obtained using the old simplified (Fig. 4A) [4] and the new improved model (Fig. 4B). The values of the maximum force are very similar, hence the same scale bar is chosen. But the maximum value using the new model is obtained in the parts of the artery that are more bended, which is more realistic and logical, since this part of the artery must be subjected to higher forces in order to restore the shape that is closer to the original and obtain better restoration of the normal blood flow. The von-Mises stress in another parameter that can be calculated based on the deformation of the arterial wall. The distribution of this quantity at the end of the simulation is shown in Fig. 5 . The scale bar is again the same, since the maximum value of stress is similar. But the change of the material model of arterial wall is even more obvious
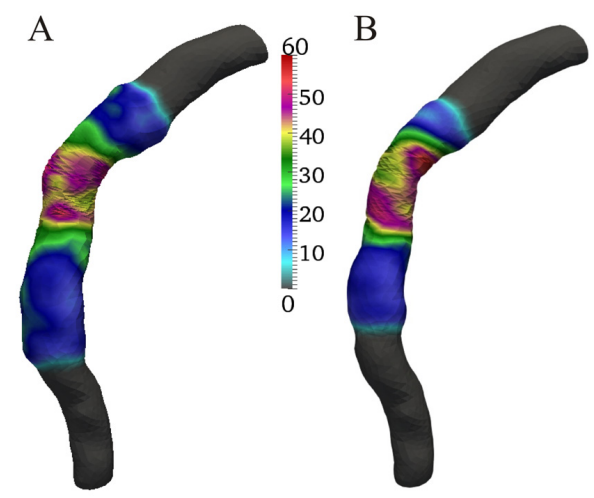

Figure 4: Distribution of the force that causes the deformation of the arterial wall;

A - simulation using the simplified numerical model; $B$-simulation using the hyperelastic numerical model; units on the scale bar are in $\mathrm{mN}$ 

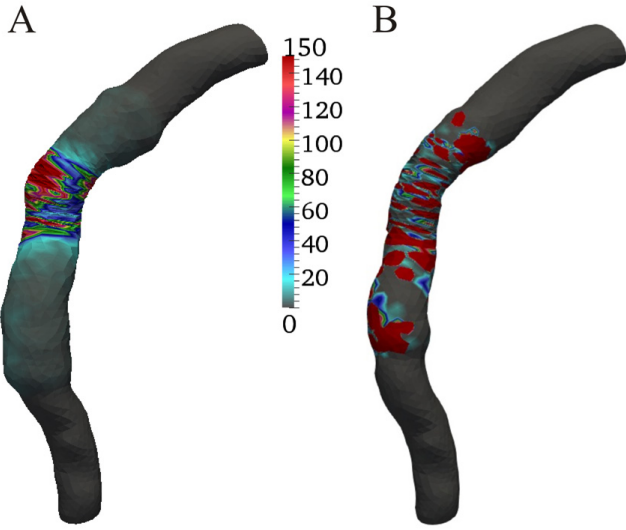

Figure 5: Distribution of the von-Mises stress, calculated based on the deformation of the arterial wall;

A - simulation using the simplified numerical model;

$B$-simulation using the hyperelastic numerical model; units on the scale bar are in $\mathrm{kPa}$

in this case, since the maximum value of stress is now located not only in the central, atherosclerotic part, where the most deformation occurs, but also in the healthy part of the artery, that opposes the deformation more intensely due to the different values of material coefficients. That is the main reason why the stent does not change significantly the shape of the healthy part of the artery in the new model, compared to the old model.

It should be noted that this numerical model is implemented in a software that takes benefits of the parallelization using GPU devices (Graphics Processing Units). More precisely, all the calculations are performed on the graphics card of the computer and this enables the simulations to run really fast. The simulation presented in this paper, with the old simplified model is executed approximately 30 seconds, while the simulation with the new improved model runs approximately 34 seconds on a PC computer with NVIDIA GeForce RTX 2080 Ti graphics card. This increase of execution time is not that significant, having in mind the benefits obtained in terms of accuracy of the simulation results.

\section{CONCLUSIONS}

The numerical simulation of the stent deployment process was already presented in literature [4]. This study presents an improvement of this numerical model, with a more realistic representation of the arterial wall as an incompressible, isotropic and hyperelastic material. The patient-specific geometrical model was used for the stent deployment simulation. The obtained results are validated against clinical data from the follow up examination by comparing the areas of the lumen along the coronary artery. The benefits of the improved model are also demonstrated through the comparison of results with the results obtained using the previous simplified version of the numerical model. The small relative and standard deviation error, obtained for the areas of the lumen, prove that this numerical model can be used to assist clinicians in decision making and treatment planning with reliable predictions of the outcome of the stent deployment procedure.

\section{ACKNOWLEDGEMENT}

The research presented in this study was part of the project that has received funding from the European Union's Horizon 2020 research and innovation programme under grant agreement No. 689068 - SMARTool. This article reflects only the author's view. The Commission is not responsible for any use that may be made of the information it contains. This work was also supported by the Ministry of Education, Science and Technological Development of the Republic of Serbia [ID 451-03-68/202014/200378]

\section{REFERENCES}

1. Gijsen, F. J., Migliavacca, F., Schievano, S., Socci, L., Petrini, L., Thury, A., Wentzel, J. J., van der Steen, A. F., Serruys, P. W., Dubini, G., (2008) Simulation of stent deployment in a realistic human coronary artery, Biomedical engineering online, 7, 23.

2. Zahedmanesh, H., John Kelly, D., Lally, C., (2010) Simulation of a balloon expandable stent in a realistic coronary artery-Determination of the optimum modelling strategy, J Biomech, 43(11), 2126-32.

3. Rebelo, N., Fu, R., Lawrenchuk, M., (2009) Study of a Nitinol Stent Deployed into Anatomically Accurate Artery Geometry and Subjected to Realistic Service Loading, J. of Materi Eng and Perform, 18, 655-663.

4. Djukic, T., Saveljic, I., Pelosi, G., Parodi, O., Filipovic, N., (2019) Numerical simulation of stent deployment within patient-specific artery and its validation against clinical data, Computer Methods and Programs in Biomedicine, 175, 121-127.

5. Karimi, A., Rahmati, S. M., Sera, T., Kudo, S., Navidbakhsh, M. (2017) A combination of experimental and numerical methods to investigate the role of strain rate on the mechanical properties and collagen fiber orientations of the healthy and atherosclerotic human coronary arteries, Bioengineered, 8:2, 154-170.

6. Larrabide, I., Kim, M., Augsburger, L., Villa-Uriol, M.C., Rüfenacht, D., Frangi, A.F., (2012) Fast virtual deployment of self-expandable stents: method and in vitro evaluation for intracranial aneurysmal stenting, Med Image Anal., 16(3), 721-730.

7. Peskin, C. S., (1977) Numerical analysis of blood flow in the heart, Journal of Computational Physics, 25(3), 220-252.

8. Paliwal, N., Yu, H., Xu, J., Xiang, J., Siddiqui, A., Yang, X., Li, H., Meng, H., (2016) Virtual stenting workflow with vessel-specific initialization and adaptive expansion for neurovascular stents and flow diverters, Comput Methods Biomech Biomed Engin., 19(13), 1423-1431. 
9. Kojic, M., Filipovic, N., Stojanovic, B., Kojic, N. (2008) Computer modeling in bioengineering: Theoretical Background, Examples and Software, Chichester, England, John Wiley and Sons.
10. Karimi, A., Navidbakhsh, M., Shojaei, A., Hassani, K., Faghihi, S. (2014) Study of plaque vulnerability in coronary artery using Mooney-Rivlin model: a combination of finite element and experimental method, Biomed Eng Appl Basis Commun, 26(01), 153-160. 